\title{
THE EFFECTIVE DESCRIPTION OF STRONG ELECTROWEAK SYMMETRY BREAKING
}

The actual mechanism of electroweak symmetry breaking remains the last unsolved part of the Standard model of electroweak interactions. We review the general requirements that all possible solutions must fulfill. Based on them the effective description of the mechanism can be formulated. We offer such a description which is a modified version of the so-called BESS model and discuss its properties.

Keywords: electroweak symmetry breaking, effective Lagrangian, BESS model.

\section{Introduction}

The gauge invariance as a fundamental principle for building the electroweak Lagrangian enables describing the electromagnetic and weak interactions under the same formalism [1]. The theory is commonly known as the Standard model of electroweak interactions (SM) [2]. The SM leads to the long list of predictions that have been successfully verified by experiments.

However, the local symmetry does not tolerate explicit mass terms for gauge fields. This seems to be in contradiction with reality in all cases but the photon. Fortunately, there is a way to reconcile the massive gauge bosons, namely $Z, W^{+}, W^{-}$, and gauge invariance: spontaneous breaking of electroweak symmetry accompanied by the Higgs mechanism. The Higgs mechanism was first suggested to explain some collective density fluctuations in plasma which were produced by a finite range electromagnetic field (a massive photon) [3]. Later it was generalized as a relativistic field theory [4] and its renormalizability was proved [5].

Despite the great success of the SM one essential component of the theory remains a puzzle: it is the actual mechanism behind the electroweak symmetry breaking (ESB). The introduction of the Higgs complex doublet scalar field of a non-zero vacuum expectation value to the electroweak Lagrangian serves as a benchmark hypothesis. A direct consequence of this hypothesis is the presence of the Higgs boson in the particle spectrum of the SM, not observed to these days. It is not the only plausible hypothesis though.

There is a host of candidates for the suitable extensions of the SM. They range from supersymmetric theories with multiple elementary Higgs bosons in their spectra to the theories of new strong interactions, like Technicolor, which might form bound states of new elementary particles in analogy with QCD. These bound states might appear in the particle spectrum as new resonances.

In more recent theories like the Little Higgs models and the Gauge-Higgs unification models, their dual-description relation to the Heavy Composite Higgs and the No Higgs strongly-interacting models has been demonstrated (see [6] and references therein). Most of these models introduce new particles at about $1 \mathrm{TeV}$.

Facing this plethora of alternative theories it is highly desirable to describe their low-energy phenomenology in a unified way. Thus, it is very useful to exploit the formalism of effective Lagrangians. Therein the so-called hidden local symmetry (HLS) approach [7] can be used to introduce new vector resonances which might originate in some new physics beyond the SM. The new particles are not only a byproduct of a particular ESB mechanism but they would be needed to tame the unitarity if the Higgs boson were not found. The HLS formalism along with the AdS/CFT correspondence plus deconstruction is also behind the dual-description relation of the recent models mentioned above [6].

In this paper we present the modified version of the existing effective model of strong ESB. The original model is called the BESS (Breaking Electroweak Symmetry Strongly) model [8]. There - beside the known SM particles - a new $S U(2)_{V}$ vector boson triplet is introduced exploiting HLS. The new triplet is supposed to represent the spin- 1 bound states of hypothetical new strong interactions. We take this model and modify its interactions of the vector triplet with fermions. In the BESS model there is a universal direct coupling of the triplet to all fermions of a given chirality. In our model, on the other hand, we admit direct couplings of the new triplet to top and bottom quarks only. The modification reflects speculations about a special role of the top quark

\footnotetext{
* Mikulas Gintner ${ }^{1}$, Josef Juran², Ivan Melo ${ }^{1}$, Beata Trpisova ${ }^{1}$

${ }^{1}$ Physics Department, University of Zilina, Slovakia, E-mail: gintner@fyzika.uniza.sk

${ }^{2}$ Institute of Experimental and Applied Physics, Czech Technical University in Prague, Czech Republic
} 
(or the third quark generation) in the mechanism of ESB. We named the modified BESS model as the top-BESS model.

In Section 2 of the paper we discuss the general requirements for the ESB mechanism which must be reflected by any ESB hypothesis. In Subsection 2.2 we briefly introduce the BESS and the top-BESS models. Besides we show the direct interaction vertices of the new vector triplet to top and bottom quarks and the differences in the couplings of the electroweak gauge bosons with these quarks. Section 3 is devoted to the phenomenology of the top-BESS model. Herein we discuss the masses and the decay widths of the new vector triplet. We also point to the forwardbackward asymmetry as a promising observable for measuring parameters of the $V$-to- $t / b$ couplings. Conclusions can be found in Section 4.

\section{The effective description of ESB}

\subsection{General requirements}

The central idea to the generating masses of the electroweak gauge bosons $W^{ \pm}$and $Z$ is the spontaneous symmetry breaking (SSB): $G \rightarrow H$ where $G$ is a global continuous symmetry of the Lagrangian and $H$ is a continuous symmetry of its vacuum (ground state), $H \subseteq G$. The Goldstone theorem [9] implies that the SSB results in appearance of massless scalar fields, the so-called NambuGoldstone bosons (NGB). The number of NGB is equal to $\operatorname{dim} G$ - $\operatorname{dim} H$, i.e. the number of "broken" generators of the group $G$. However, once $F \subset G$ is made local by introducing gauge fields interacting with the NGB's the corresponding NGB's disappear from the spectrum while the $G-H$ gauge fields become massive and acquire longitudinal degrees of freedom. This is depicted in the following figure:

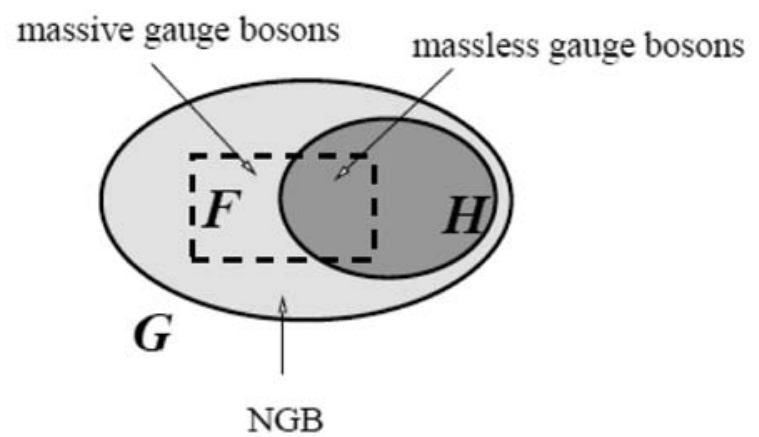

Now we formulate the conditions that must be fulfilled in order to build the most general effective Lagrangian describing the Higgs mechanism that provides masses for the $Z^{0}$ and $W^{ \pm}$ gauge bosons [10]:

- A part of the SM must exhibit the SSB pattern $G \rightarrow H$, where $G$ is a global symmetry and $H$ is its subgroup. Since we need masses for three gauge bosons we require that there would be at least 3 broken generators: $\operatorname{dim} K \geq 3$ where $K=G / H$ $(\operatorname{dim} K=\operatorname{dim} G-\operatorname{dim} H)$.

- Three of broken generators must generate a local symmetry. The massive gauge bosons must be coupled to the corresponding three NGB's.

- The gauge group $S U(2)_{L} \times U(1)_{Y}$ has to be contained in $G$. The photon is a massless gauge boson thus $U(1)_{e m} \subset H$. Therefore $\operatorname{dim} G \geq 4, \operatorname{dim} H \geq 1$.

- If the SSB bears the responsibility for the masses of gauge bosons then the masses should be given by the scale $v$ of the SSB, $M_{W, Z}=\mathcal{O}(v)$. The value of $v$ can be obtained from the Fermi constant $G_{F}$ which is measured in the muon decay:

$$
\begin{aligned}
& v^{2}=\frac{1}{\sqrt{2} G_{F}}, \quad G_{F} \approx 1.17 \times 10^{-5} \mathrm{GeV}^{-2} \\
& \Longrightarrow v \approx 246 \mathrm{GeV}
\end{aligned}
$$

- The electrowek gauge symmetry predicts that the relative strength of the charged weak currents to the neutral weak currents

$$
\rho=\frac{\text { charged current }}{\text { neutral current }}=\frac{M_{W}^{2}}{M_{Z}^{2} \cos \theta_{W}}
$$

is equal to 1 at tree level. Generally, at loop levels the gauge symmetry $S U(2)_{L} \times U(1)_{Y}$ admits large corrections to $\rho$. Nevertheless, when the $H$ group contains the so-called custodial symmetry $S U(2)_{V}$ the radiative corrections to the $\rho$ parameter must be small. When measured in experiments the $\rho$ parameter comes up with the value

$$
\rho_{\exp }=1+\mathcal{O}\left(10^{-2}\right)
$$

which supports the presence of the custodial symmetry. The condition $S U(2)_{V} \subset H$ implies $\operatorname{dim} H \geq 3$.

If we consider $K=3$ (otherwise we would have to explain where the uneaten NGB's are) the constraints listed above lead to a unique choice of the groups $G$ and $H$ :

$$
\begin{aligned}
& G=S U(2)_{L} \times S U(2)_{R}, \\
& H=S U(2)_{V}, K=S U(2)_{A},
\end{aligned}
$$

where $S U(2)_{V}$ is a diagonal subgroup of $S U(2)_{L} \times S U(2)_{R}$ and $S U(2)_{A}$ is its axial ") "subgroup". This also implies that NGB's are pseudoscalar particles.

Let us mention that the gauge invariance under a chiral symmetry group excludes explicit mass terms for fermions. However, gauge invariant mass terms for fermions can be generated through the ESB mechanism.

\footnotetext{
${ }^{1)} S U(2)_{A}$ is not a group even though it is often called that way in the physical literature.
} 


\subsection{From BESS to top-BESS}

The BESS model [8] effectively describes a Higgsless ESB mechanism accompanied by a hypothetical strong triplet of vector resonances $\tilde{V}_{\mu}^{\alpha}$. In the HLS formulation the symmetry of the model is $\left[S U(2)_{L} \times S U(2)_{R} \times U(1)_{B-L}\right]^{\text {glob }} \times S U(2)_{V}^{l o c}$ where $B$, $L$ are the baryon and lepton numbers, respectively. The hypothetical vector triplet is introduced as the $S U(2)_{V}$ gauge bosons. Beside the indirect interactions of the new vector resonances to the SM fermions induced by the mixing of $\widetilde{V}_{\mu}^{\alpha}$ with the SM gauge bosons, the BESS model couples the vector resonances directly and universally to all SM fermion generations of a given chirality. The direct interactions are parameterized by two free parameters, $b$ for the left fermions, and $b^{\prime}$ for the right fermions. The low energy measurements imply the following limits: $b \lesssim 0.01, b^{\prime} \approx 0$.

In the top-BESS model we modify the fermion sector by considering no direct interactions of $V$ to fermions other than $t$ and $b$. The $S U(2)_{L}$ symmetry does not allow us to disentangle the $V$-to- $t_{L}$ coupling from the $V$-to- $b_{L}$ one. However, it can be done in the case of right fermions. We do it and to simplify the numerical analysis of the model we turn off the direct interaction of $V$ to $b_{R}$ completely. The full form of the top-BESS Lagrangian can be found in [11]. Here, we show only the part responsible for the direct interactions of $t$ and $b$ quarks to the new vector resonances parameterized by the $b_{1,2}$ coupling constants as well as the nonSM interactions of $t$ and $b$ with the SM gauge bosons

$$
\begin{aligned}
& \left(\begin{array}{l}
\tilde{W}^{ \pm} \\
\tilde{V}^{ \pm}
\end{array}\right)=\left[\left(\begin{array}{cc}
1 & -\sqrt{\alpha} x \\
\sqrt{\alpha} x & 1
\end{array}\right)+\mathcal{O}\left(x^{2}\right)\right]\left(\begin{array}{l}
W^{ \pm} \\
V^{ \pm}
\end{array}\right), \\
& x=\frac{\bar{M}_{W}}{\bar{M}_{V}}=\frac{1}{\sqrt{\alpha}} \frac{g}{g^{\prime \prime}}
\end{aligned}
$$

where $G=\sqrt{g^{2}+g^{\prime 2}}, c_{W}=\bar{M}_{V} / \bar{M}_{Z}, s_{W}=\sqrt{1-c_{W}^{2}}, c_{2 W}=$ $=c_{W}^{2}-s_{W}^{2}, s_{2 W}=2 s_{W} c_{W}, \bar{M}_{V}=g v / 2, \bar{M}_{Z}=G v / 2$. The free parameter $\alpha$ is related to the mass of the vector triplet, degenerated in the leading order approximation: $\bar{M}_{v}=\sqrt{\alpha} g^{\prime \prime} v / 2 \cdot \bar{M}_{W}$ and $\bar{M}_{Z}$ are the $W$ and $Z$ boson masses in the leading order approximation, respectively.

The measurement of $Z b \bar{b}$ vertex constrains the $V$-to- $t_{L}$ coupling to relatively small values. Due to the disentangling of the $t_{R}$ interactions with $V$ from the $b_{R}$ interactions with $V$ the measurement does not limit the $V$-to- $t_{R}$ interaction. For the low-energy limits on the model's parameters, see $[11,12]$.

\section{Phenomenology of the top-BESS model}

\subsection{Masses and widths of the new vector resonance triplet}

The masses of the $S U(2)_{V}$ neutral and charged resonances depend on the parameters $\alpha$ and $g^{\prime \prime}$ as shown in Fig. 1. In all cal-

$$
\begin{aligned}
L_{\rho}^{(t, b)_{L}} & =\frac{b_{1}}{1+b_{1}} g \bar{\psi}_{L} \tilde{W}^{a} \tau_{a} \psi_{L}+\frac{b_{1}}{1+b_{1}} \frac{g^{\prime \prime}}{2} \bar{\psi}_{L} \tilde{V}^{a} \tau_{a} \psi_{L} \\
& =\frac{1}{2} \frac{b_{1} g}{1+b_{1}}\left(\bar{t}_{L} \tilde{W}^{3} t_{L}-\bar{b}_{L} \tilde{W}^{3} b_{L}\right)+\frac{1}{\sqrt{2}} \frac{b_{1} g}{1+b_{1}}\left(\bar{t}_{L} \tilde{W}^{+} b_{L}-\bar{b}_{L} \tilde{W}^{-} t_{L}\right) \\
& =\frac{1}{2} \frac{b_{1}}{1+b_{1}} \frac{g^{\prime \prime}}{2}\left(\bar{t}_{L} \tilde{V}^{3} t_{L}-\bar{b}_{L} \tilde{V}^{3} b_{L}\right)+\frac{1}{2} \frac{b_{1}}{1+b_{1}} \frac{g^{\prime \prime}}{2}\left(\bar{t}_{L} \tilde{V}^{+} b_{L}-\bar{b}_{L} \tilde{V}^{-} t_{L}\right) \\
L_{\rho}^{(t, b)_{R}} & =\frac{b_{2}}{1+b_{2}} g^{\prime}\left(\bar{\psi}_{R} P_{0}\right) \not B \tau_{3}\left(P_{0} \psi_{R}\right)+\frac{b_{2}}{1+b_{2}} \frac{g^{\prime \prime}}{\sqrt{2}}\left(\bar{\psi}_{R} P_{0}\right) \tilde{V}^{3} \tau_{3}\left(P_{0} \psi_{R}\right) \\
& =\frac{1}{2} \frac{b_{2} g}{1+b_{2}}\left(\bar{t}_{R} \not B t_{R}\right)+\frac{1}{2} \frac{b_{2}}{1+b_{2}} \frac{g^{\prime \prime}}{\sqrt{2}}\left(\bar{t}_{R} \tilde{V}^{3} t_{R}\right)
\end{aligned}
$$

where $\psi=(t, b)^{T}$, and $P_{0}=\operatorname{diag}(1,0)$. The gauge couplings $g, g^{\prime}$ and $g^{\prime \prime}$ correspond to the $S U(2)_{L}, U(1)_{Y}$, and $S U(2)_{V}$ gauge symmetry groups of the model, respectively. As a consequence of turning off the $V$-to- $b_{R}$ coupling in (2) neither $t_{R}$ couples directly to the charged $V$-resonance.

The Lagrangians (1) and (2) are expressed in terms of gauge field flavor eigenstates. However, in experiments we measure the mass eigenstates. The relations between the two sets of the gauge field eigenstates are given by culations depicted here and below we consider the fixed values of the $Z$ and $W$ masses as taken from experiment. To compensate for the non-SM corrections to $M_{Z}$ and $M_{W}$ the SM parameters $v, g$, and $g^{\prime}$ vary slightly with $g^{\prime \prime}$.

The vector resonances decay dominantly to the SM gauge bosons and/or to the third generation of quarks. In Fig. 2 we show the total decay widths of the vector resonances [13]. The plots of the partial decay widths are shown in Fig. 3. Finally, we compare the BESS and the top-BESS models in terms of the total decay

$$
\left.\left(\begin{array}{c}
\tilde{W}^{3} \\
\not B \\
\tilde{V}^{3}
\end{array}\right)=\left[\begin{array}{ccc}
s_{W} & c_{W} & -c_{W} \sqrt{\alpha} y \\
c_{W} & -s_{W} & -s_{W} \sqrt{\alpha} y \\
s_{2 W} \sqrt{\alpha} y & c_{2 W} \sqrt{\alpha} y & 1
\end{array}\right)\right]+\mathcal{O}\left(y^{2}\right)\left(\begin{array}{c}
A \\
Z \\
V^{0}
\end{array}\right), \quad y=\frac{\bar{M}_{Z}}{\bar{M}_{V}}=\frac{1}{\sqrt{\alpha}} \frac{G}{g^{\prime \prime}}
$$



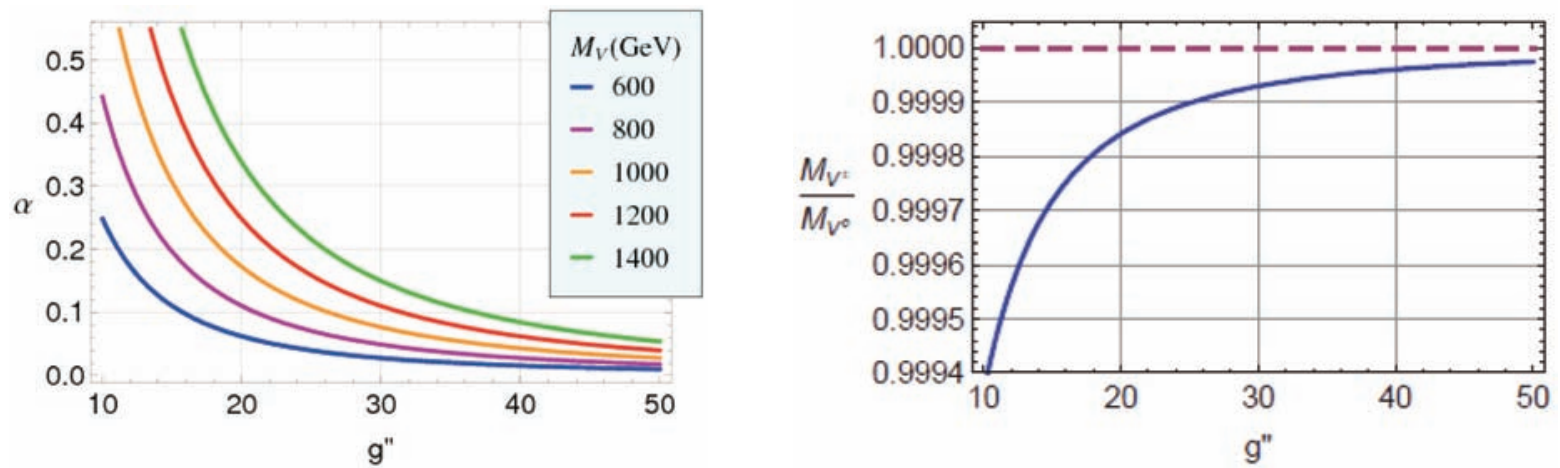

Fig. 1 At the left: The relations between $a$ and the $S U(2)_{V}$ gauge coupling $g^{\prime \prime}$ at various fixed masses of $V$. At the right: The splitting of the $M_{V^{ \pm}}-t o-M_{V^{0}}$ degeneracy as a function of $g^{\prime \prime}$.
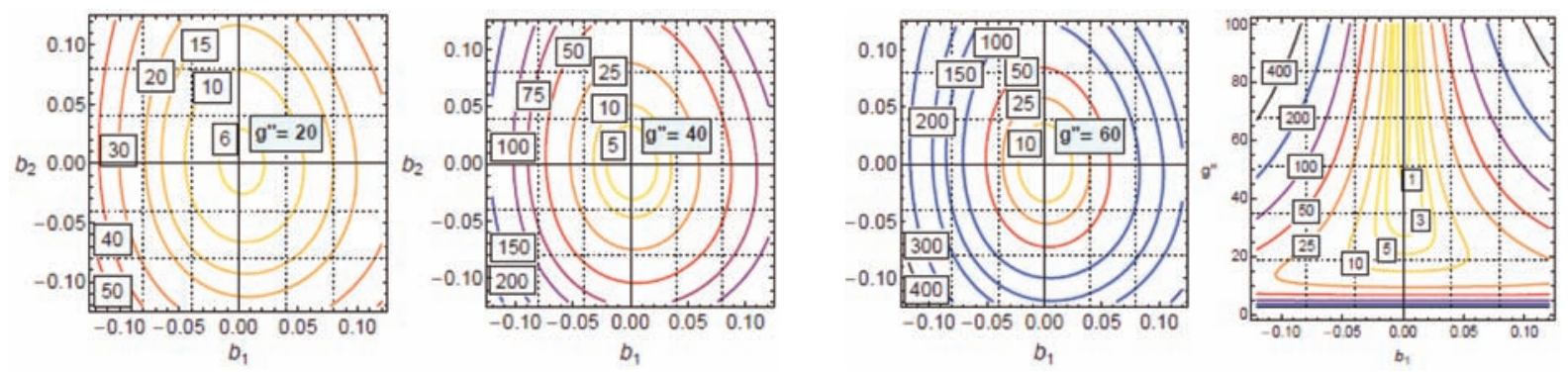

Fig. 2 The top-BESS total decay width contours of $V^{0}$ in the $\left(b_{1}, b_{2}\right)$ parametric space at various values of $g^{\prime \prime}$, and of $V^{ \pm}$ in the $\left(b_{1}, g^{\prime \prime}\right)$ parametric space. The contour labels are in $\mathrm{GeV}$.
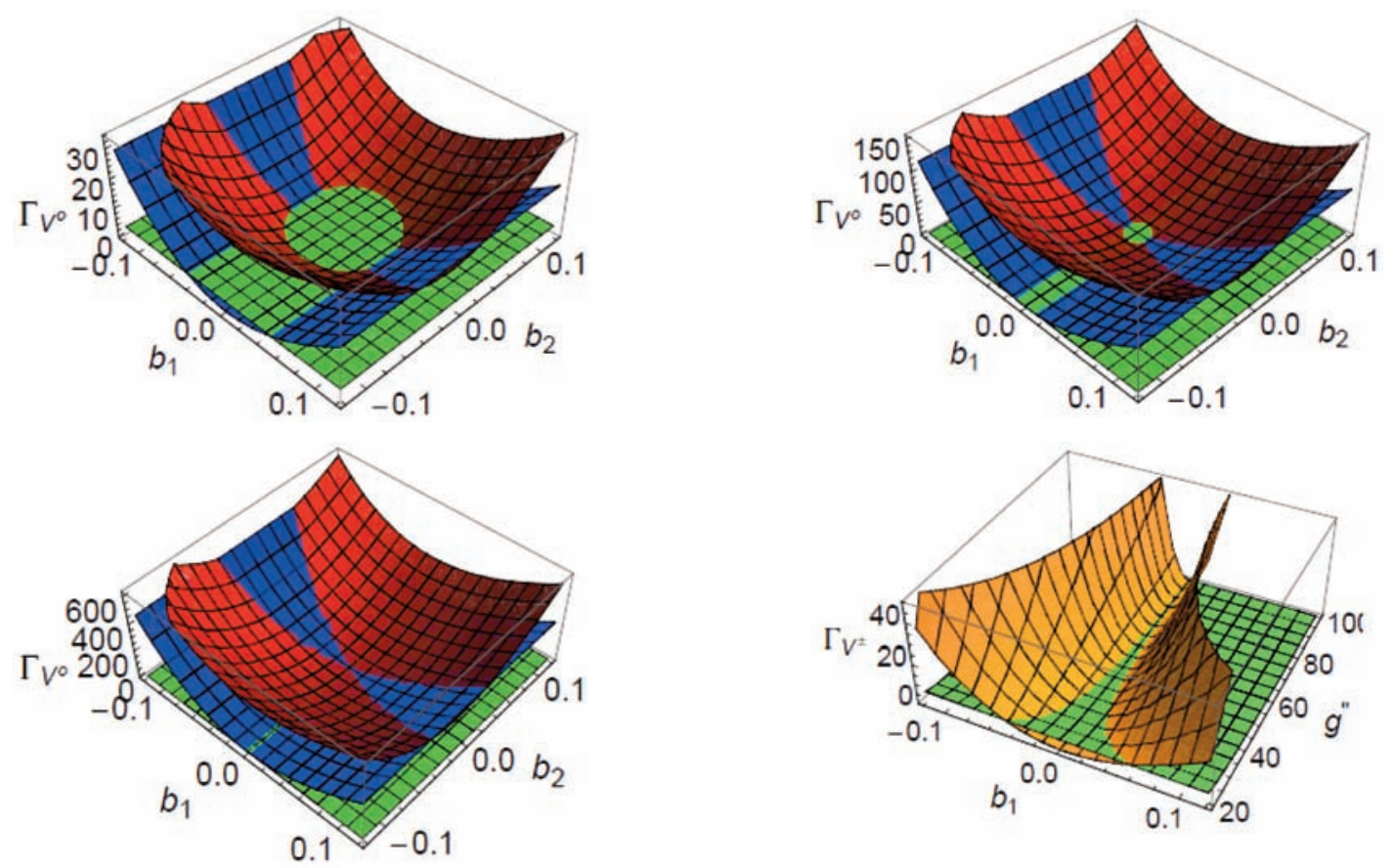

Fig. 3 The top-BESS partial decay widths of $V^{0}$ as functions of $b_{1}$ and $b_{2}$ at $g^{\prime \prime}=25,50,100$, from the left to the right, respectively, and of $V^{+}$as functions of $b_{1}$ and $g^{\prime \prime}$. For the neutral resonance, the green, blue, red surface corresponds to the $W^{+} W^{-}, b \bar{b}$, and $\bar{t}$ channel, respectively. For charged resonance the green, orange surface corresponds to the $W^{+} Z$ and $t \bar{b}$ channel, respectively. 

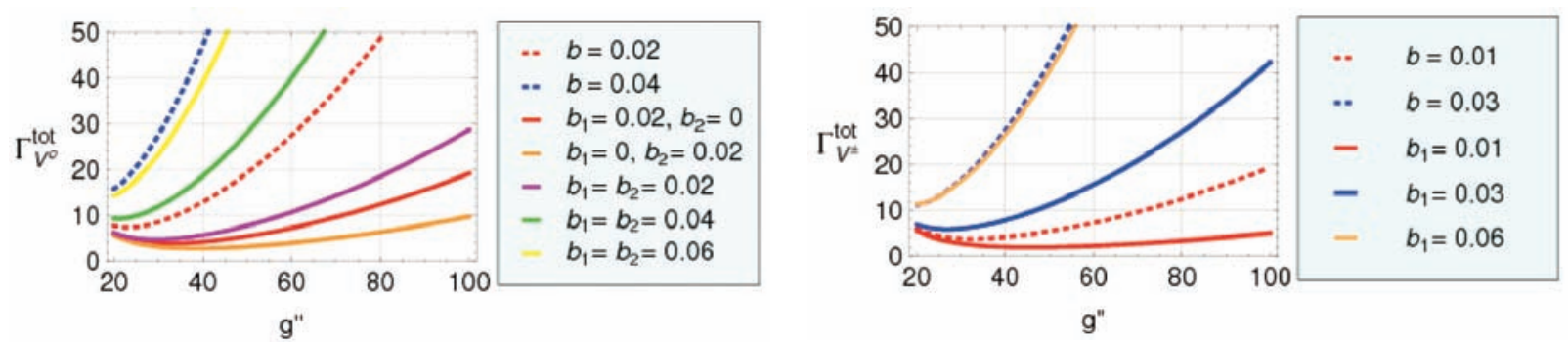

Fig. 4 Differences between the BESS model (dotted lines) and the top-BESS model (solid lines) demonstrated through the total decay widths of $V^{0}$ and $V^{ \pm}$(in $\mathrm{GeV}$ ).

widths of $V^{0}$ and $V^{ \pm}$in Fig. 4 [13]. In all cases we assume $M_{V^{0}}=$ $=1 \mathrm{TeV}$.

\subsection{Experimental signatures}

It would seem natural to search for the signs of the new resonances in the processes where the electroweak gauge bosons and/or the $t$ and $b$ quarks are involved. At the Large Hadron Collider (LHC) this is often a difficult case due to the large backgrounds to the processes with $b$ and $t$ in the final state and/or due to the negligible $b$ and $t$ luminosities in $p p$ collisions.

In the BESS model the new $S U(2)_{V}$ vector bosons mix with the electroweak gauge bosons. While the mixing complicates theoretical analysis of the model it might provide some advantages in experimental searches. The mixing generates interactions of the new resonances with the fermions of lighter generations. Although these interactions are suppressed by the mixing factors, the LHC processes enabled by them have the advantage of higher parton luminosities and more favorable final state topologies. In [12] we have calculated sensitivities of the $p p \rightarrow \overline{t t} X, b \bar{b} X, t \bar{b} X, W^{+} W^{-} X$, $W^{ \pm} Z X$ processes with subsequent decays to leptons and hadronic jets at the LHC without considering backgrounds. At this level of analysis the investigated processes have displayed promising potential to serve as probes of new physics under consideration. Nevertheless, further and more detailed investigation of the issues is necessary.

New ESB-responsible physics can also be studied at a future $e^{+} e^{-}$collider under preparation which is known under its generic name of ILC (International Linear Collider). While the LHC will serve as a discovery machine the ILC will provide much cleaner signal to measure parameters of new physics. In [14] we have analyzed three ILC processes $-e^{+} e^{-} \rightarrow \bar{t} \bar{t}, e^{+} e^{-} \rightarrow b \bar{b}$, and $e^{+} e^{-} \rightarrow$ $W^{+} W^{-}-$at the collision energies $800-1200 \mathrm{GeV}$ as probes of the top-BESS model with $M_{V^{0}}=1 \mathrm{TeV}$.

The above mentioned investigations focused on discovering and distinguishing mass peaks of the $V$-resonances in the invariant mass distributions of the $V$ decay products. The observation of a mass peak would result in determining the mass and, perhaps, the width of the observed resonance. However, here we would like to point to yet another ILC observables which might be suitable for determining the values of $b_{1}$ and $b_{2}$ parameters. Namely, they are the forward-backward assymmetries of top and bottom quarks. This is illustrated in Figs. 5 and 6 for processes $e^{+} e^{-} \rightarrow t_{t}$ and $e^{+} e^{-} \rightarrow b \bar{b}$, respectively, with $M_{V^{0}}=1 \mathrm{TeV}$. The curves for the forward-backward asymmetries depend significantly on the values

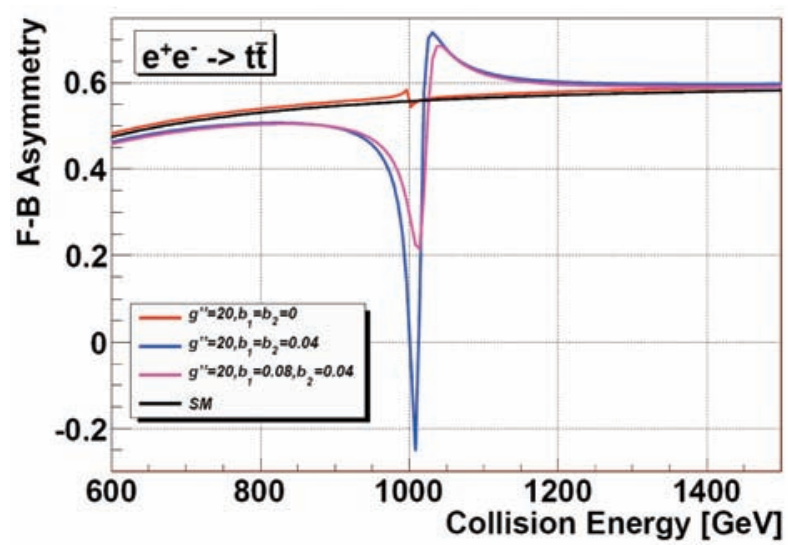

Fig. 5 The forward-backward asymmetry for the process $e^{+} e^{-} \rightarrow$ tt as a function of the collision energy when $M_{V^{0}}=1 \mathrm{TeV}$. The sensitivity of the SM curve to the Higgs mass is negligible.

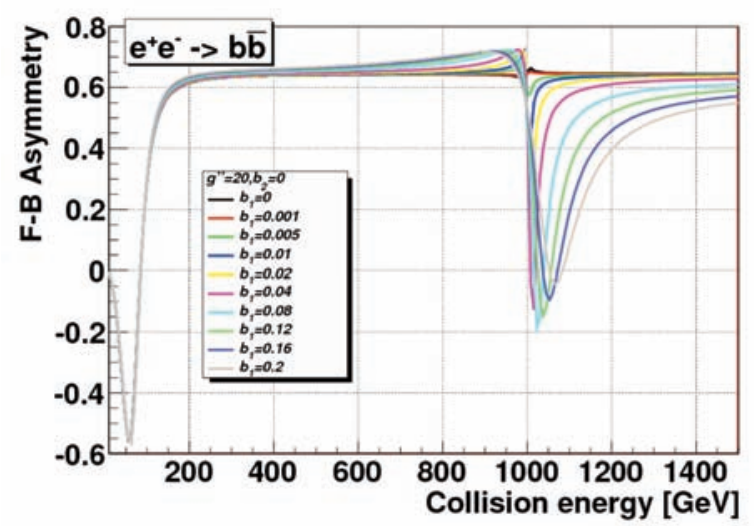

Fig. 6 The forward-backward asymmetry for the process $e^{+} e^{-} \rightarrow b \bar{b}$ as a function of the collision energy when $M_{V^{0}}=1 \mathrm{TeV}$. While $b_{2}=0$ the $b_{1}$ parameter runs through 0 to 0.2 . 
of the $b_{1,2}$ parameters. They also differ significanly from the SM curve in the region around the mass of the $V$ resonance.

Regarding hadron colliders the forward-backward asymmetry is not applicable at the LHC because the symmetry of the colliding proton beams wipes up the forward-backward asymmetry. On the other hand, the Tevatron collider is free of this problem because it collides protons with antiprotons. However, its collision energy is probably too low to probe $1 \mathrm{TeV}$ resonances.

\section{Conclusions}

In this paper we have presented the status of our ongoing study of the top-BESS model which is a well-motivated modifica- tion of the original BESS model. We had introduced the modifications to obtain the effective description of a certain class of theories which aim at explaining the mechanism of ESB. These are the theories with the top quark playing a special role in the ESB mechanism.

It appears that the properties of the theories representable by the top-BESS model can be studied at both the LHC as well as ILC colliders. While the $S U(2)_{V}$ resonance triplet can be discovered at the LHC by observing the invariant mass distributions of its decay products the detailed properties of the resonances can be studied at the ILC. For example, the observation of the forwardbackward asymmetry at the ILC is well suited for the measurement of the $b_{1,2}$ parameters. Nevertheless, further investigation of the related issues is needed. This work is in progress.

\section{References}

[1] YANG, C. N., MILLS, R. L.: Phys. Rev. 96, 1954, 191.

[2] GLASHOW, S. L.: Nucl. Phys. 22, 1961, 579; SALAM, A., WARD, J. C.: Phys. Lett. 13, 1964, 168; WEINBERG, S.: Phys. Rev. Lett. 19, 1967, 1264.

[3] ANDERSON, P. W.: Phys. Rev. 130, 1963, 439.

[4] ENGlert, F., Brout, R.: Phys. Rev. Lett. 13, 1964, 321; HIGGS, P. W.: Phys. Rev. Lett. 12, 1964, 132; Phys. Rev. Lett. 13, 1964, 508; Phys. Rev. 145, 1966, 145.

[5] HOOFT, G.: Nucl. Phys. B35, 1971, 167.

[6] CHENG, H-C.: Proceedings of 15th International Conference on Supersymmetry and the Unification of Fundamental Interactions (SUSY07), Karlsruhe, 2007, hep-ph/0710.3407.

[7] BANDO, M., KUGO, T., YAMAWAKI, K.: Phys. Rep. 164, 1988, 217.

[8] CASAlBUONI, R., de CURTIS, S., DOMINICI, D., GATO, R.: Phys. Lett. B155, 1985, 95; CASALBUONI, R., de CURTIS, S., DOMINICI, D., GATO, R.: Nucl. Phys. B282, 1987, 235; CASALBUONI, R., CHIAPPETTA, P., de CURTIS, S., FERUGLIO, F., GATTO, R., MELE, B., TERRON, J.: Phys. Lett. B249, 1990, 130.

[9] GOLDSTONE, J.: Nuovo Cimento 19, 1961, 154; GOLDSTONE, J., SALAM, A., WEINBERG, S.: Phys. Rev. 127, $1962,965$.

[10] DOBADO, A., GOMEZ-NICOLA, A., MAROTO, A. L., PELAEZ, J. R.: Effective Lagrangians for the Standard Model, Springer 1997, ISBN 3-540-62570-4.

[11] GINTNER, M., MELO, I., TRPISOVA., B.: Acta Phys. Slov. 56, 2006, 473-483.

[12] GINTNER, M., MELO, I., TRPISOVA., B.: Proceedings of $16^{\text {th }}$ Conference of Czech and Slovak Physicists, Hradec Kralove, 2007; Proceedings of $10^{\text {th }}$ Small Triangle Meeting on Theoretical Physics, Medzilaborce, 2008, arXiv:0903.1981.

[13] GINTNER, M., JURAN, J., MELO, I.: The proceedings of The 2009 Europhysics Conference on High Energy Physics, July 16-22, 2009, Krakow, Poland.

[14] GINTNER, M., JURAN, J., MELO, I., TRPISOVA., B.: Proceedings of 17th Conference of Slovak Physicists, Bratislava, 2009. 